\title{
Propiedades dieléctricas de pastas de cemento con reducido contenido en agua libre
}

\author{
J. DE FRUTOS 1 , L. SOLER ${ }^{2}$, C. ANDRADE ${ }^{3}$ \\ 1Dpto. Física Aplicada. ETSIT-UPM. Ciudad Universitaria. 28040 Madrid(Spain) \\ ${ }^{2}$ CIEMAT. Ciudad Universitaria. 28040 Madrid (Spain) \\ ${ }^{3}$ Instituto E. Torroja - C.S.I.C. - P.O. Box 19002. 28080 Madrid (Spain)
}

\begin{abstract}
En el presente trabajo se estudia como varían las propiedades dieléctricas del material con su contenido en humedad de pastas de cemento, con y sin cloruros, mediante la Espectroscopia de Impedancia Electroquímica, EIS. Para ello se fabricaron pastas de cemento con diferentes relaciones agua/cemento, y con diferente contenido de cloruros. Después de someter las muestras a procesos de secado, se procede a la rehidratación de las mismas, controlando el contenido de agua, por medio del incremento en peso relativo. Se hace un estudio pormenorizado de la respuesta eléctrica del material en el rango de $20 \mathrm{~Hz}$ a $40 \mathrm{MHz}$, en función del contenido de agua, y de la presencia de cloruros, y se relacionan los resultados con los procesos químicos presentes en el material.
\end{abstract}

Palabras Clave: Hormigón, cemento, constante dieléctrica, Espectroscopía de Impedancia Electroquímica, contenido de agua.

\section{Dielectric properties of concrete paste with low content of free water}

In this work the dielectric properties of concrete with and without chlorides as a function of their water contents were studied by the Electrical Impedance Spectroscopy (EIS). Specimens of cement paste with different water/cement ratio and chlorides content was analyzed. After drying the specimens, the rehydratation was controled by the relative weight increasing. A detailed study of the dielectric behavior in the range of $20 \mathrm{~Hz}$ to $40 \mathrm{MHz}$ according to the water and the chlorides contents was made. The results were discussed in the framework of the material processes.

Keywords: Concrete, water content, dielectric constant, Electrochemical Impedance Spectroscopy (EIS)

\section{INTRODUCCIÓN}

El hormigón es un material poroso que presenta la posibilidad de almacenar en sus poros cantidades relativas de agua grandes sin que se altere significativamente su resistencia mecánica, aunque sí se ven afectadas muchas otras propiedades que tienen que ver con su durabilidad. Así, aspectos como fluencia, retracción o resistencia a la penetración de sustancias agresivas dependen del contenido en humedad del hormigón. Por otra parte, el agua es uno de los constituyentes fundamentales del hormigón. La presencia en los poros de este elemento, puede causar reequilibrios composicionales, y modificar la proporción inicial de agua/cemento.

Eléctricamente, el hormigón es un material que se comporta como dieléctrico o como conductor en función de su contenido en humedad. El hormigón húmedo tiene una resistividad alrededor de $50 \Omega \mathrm{m}$ a $1 \mathrm{KHz}$, mientras que cuando está completamente seco, la resistividad alcanza niveles del orden de $10^{9} \Omega \mathrm{m}(1)$.

Durante los años 60-70 se publicaron los primeros intentos de medida del contenido en agua del hormigón (2-4) a partir de la medida de su constante dieléctrica, en frecuencias del orden de MHz-GHz. También se midió para estudiar la evolución del fraguado del cemento (3) y más recientemente se está utilizando para caracterizar los hormigones de altas prestaciones y los libres de defectos (MDF) (5) que son hormigones que pretenden competir en aplicaciones con algunas cerámicas.

En trabajos anteriores $(6,7)$ se inició el estudio por medio de espectroscopia de Impedancia Electroquímica de hormigones, tratando de relacionar la constante dieléctrica de estas muestras con la frecuencia, contenido de agua, contenido de cloruros y porosidad del material. Como resultado de este estudio, se puso de manifiesto, que a igual presión de vapor de agua (humedad relativa) adquieren más rápidamente y en mayor proporción agua las muestras con cloruros, al tener un tamaño de poro menor. Por otra parte, la constante dieléctrica del hormigón secado a $105^{\circ} \mathrm{C}$ varía con la frecuencia mucho menos que la variación registrada cuando el contenido en agua aumenta. Al mismo tiempo, este parámetro, es muy dependiente de la frecuencia, obteniéndose valores muy grandes a bajas frecuencias, y aproximándose a los valores teóricos de caracterización (rango de microondas) para frecuencias altas. Lo que hace imprescindible asociar el dato de constante dieléctrica con la frecuencia de medida. Confirmamos que la constante dieléctrica aumenta con el contenido en agua de los poros del hormigón para todas las frecuencias.

En este trabajo, pretendemos dar un paso más en el conocimiento de estos materiales y su caracterización por medio de impedancia electroquímica. Los procesos que involucran movimiento de agua proporcionan respuesta diferente en función del contenido inicial en el proceso de amasado, la porosidad inducida, y el contenido de cloruros presente en el mismo. Estudiaremos el comportamiento eléctrico de estas pastas en procesos de rehidratación, tratando de discriminar la parte de 
agua que se retiene en los poros del material del agua, y el contenido de agua que pasa a formar parte constituyente de la pasta.

\section{PROCEDIMIENTO EXPERIMENTAL}

Se han preparado materiales de tres tipos:

$$
\begin{aligned}
& \text { cemento }(100 \mathrm{gr})+\mathrm{H}_{2} \mathrm{O}(50 \mathrm{gr}) \\
& \text { cemento }(100 \mathrm{gr})+\mathrm{H}_{2} \mathrm{O}(50 \mathrm{gr}) \\
& +\mathrm{CaCl}_{2} 2 \mathrm{H}_{2} \mathrm{O}(2,56 \mathrm{gr}) \\
& \text { cemento }(100 \mathrm{gr})+\mathrm{H}_{2} \mathrm{O}(40 \mathrm{gr})
\end{aligned}
$$

(Serie A)

(Serie B)

(Serie C)

Las tres familias fueron sometidas a un proceso de curado durante 10 días en condiciones de $100 \%$ de Humedad Relativa, H.R., y $22{ }^{\circ} \mathrm{C}$ y posteriormente fueron mecanizadas en paralelepípedos de $2.5 \mathrm{~mm}$ de espesor y $600 \mathrm{~mm}^{2}$ de superficie y electrodadas con pintura de plata de secado a temperatura ambiente.

A continuación, las muestras fueron sometidas a un proceso de secado a $100{ }^{\circ} \mathrm{C}$ durante 48 horas, a partir del cual, una parte de ellas se introdujeron en silicona para bloquear la entrada de agua, y el resto se mantuvieron en una atmósfera de humedad controlada (33\% H.R.), y en intervalos de 4, 8, 12, 24, 48, 72 y 112 horas se van sacando e introduciendo en silicona. De esta manera, se pretendía disponer para cada familia de una gama de muestras con diferentes contenidos de humedad. No obstante, al repetir medidas para verificar resultados, se puso de manifiesto, que aunque la silicona retarda la entrada de agua en las muestras, en un periodo de tiempo relativamente corto (10-15 días), las muestras adquieren todas un nivel de humedad equivalente. Para solventar este problema, se opta por caracterizar las muestras en función, no de la atmósfera a que se somete, sino del aumento proporcional en peso de la muestra. Esto dificulta el proceso de medi$\mathrm{da}$, porque exige realizar las medidas en un periodo de tiempo corto, para evitar que la muestra cambie sus propiedades.

Las muestras así conseguidas, fueron caracterizadas eléctricamente, en función de la frecuencia y a temperatura constante, por medio de un puente HP-4284A Precision LCR Meter de la casa Hewlett-Packard, en el rango de 20 a $10^{6} \mathrm{~Hz}$, utilizando el proceso de compensación de impedancia, y con una tensión de medida de 1 voltio.

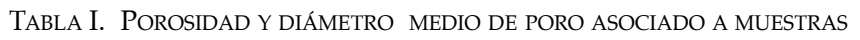
DE LAS TRES SERIES

\begin{tabular}{|c|l|c|c|c|c|}
\hline Muestra & Parámetro medido & \multicolumn{3}{|c|}{ Valores } & Porosidad Total (\%) \\
\hline \multirow{2}{*}{$\mathrm{A}$} & Diámetro medio $(\mu \mathrm{m})$ & 0.4 & 0.11 & & \\
\cline { 2 - 6 } & Porosidad asociada (\%) & 5 & 13 & & 18 \\
\hline \multirow{2}{*}{$\mathrm{B}$} & Diámetro medio $(\mu \mathrm{m})$ & 10 & 0.07 & 0.02 & \\
\cline { 2 - 6 } & Porosidad asociada (\%) & 4.5 & 10.7 & 5.9 & 21 \\
\hline \multirow{2}{*}{$\mathrm{C}$} & Diámetro medio $(\mu \mathrm{m})$ & 0.3 & 0.08 & & \\
\cline { 2 - 6 } & Porosidad asociada (\%) & 4.5 & 7.5 & & 12 \\
\hline
\end{tabular}

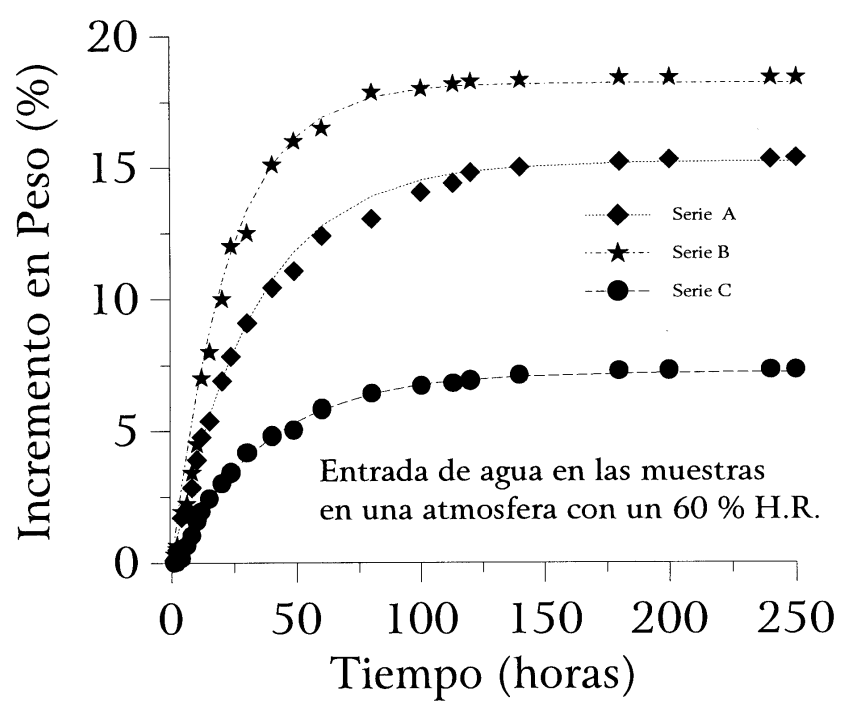

Figura 1. Incremento en peso del contenido de agua en las muestras con el tiempo, en una atmósfera de $60 \%$ de H.R.

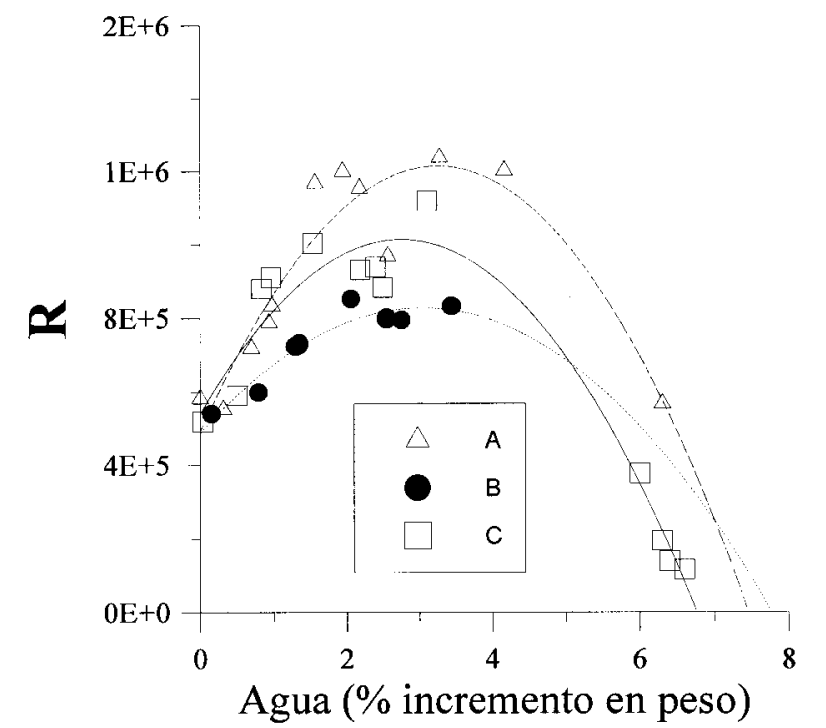

Figura 2. Variación de la impedancia real (R) a $1 \mathrm{kHz}$ con el contenido de agua

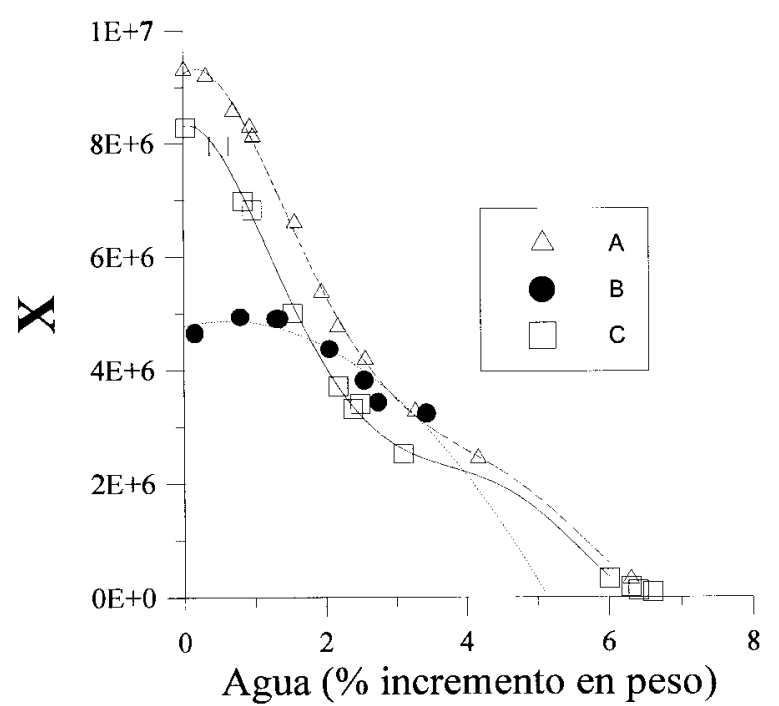

Figura 3. Variación de la parte imaginaria de la impedancia (-X) a 1 $\mathrm{kHz}$ con el contenido de agua. 


\section{RESULTADOS Y DISCUSIÓN}

Uno de los aspectos más determinantes en la entrada de agua en las muestras, es su grado y distribución de porosidad. Se hizo un estudio de este parámetro para las diferentes familias por medio de un porosímetro de mercurio. Los aspectos más destacados de este estudio son los que se recogen en la tabla I.

Los resultados anteriores, ponen de manifiesto las fuertes diferencias de porosidad originadas en este tipo de materiales, dependiendo del procesado. Las muestras A y $\mathrm{C}$, que únicamente difieren en la relación inicial a/c, presentan dos familias de poros, en regiones muy próximas, con un aumento considerable de la porosidad en las muestras A. La variación más notable la encontramos con las muestras B. La presencia de cloruros, modifica fuertemente la porosidad en el material, presentando un porcentaje relativamente alto de poros de gran tamaño, y de forma similar a los otros procesados, presenta

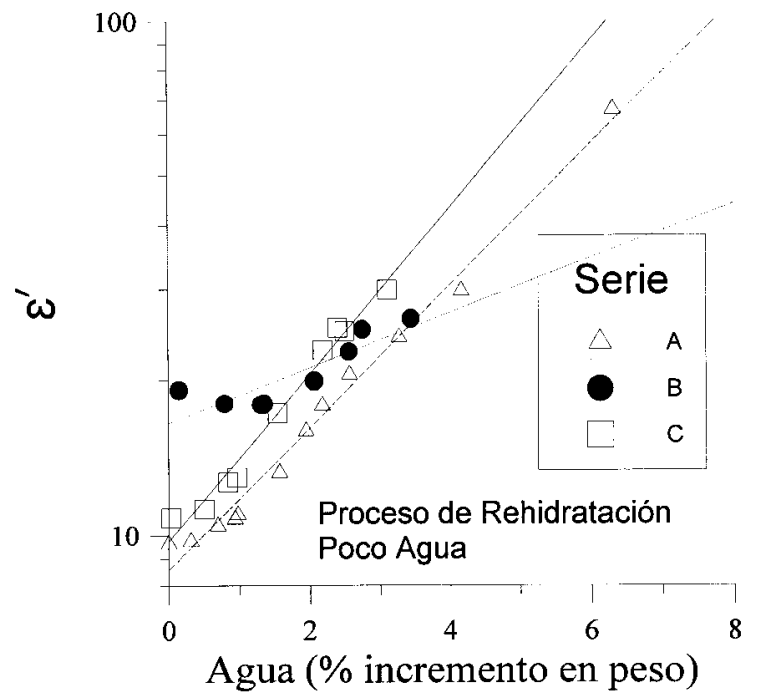

Figura 4.Variación con el contenido de agua a $1 \mathrm{kHz}$, de la parte real de la constante dieléctrica $\left(\varepsilon^{\prime}\right)$

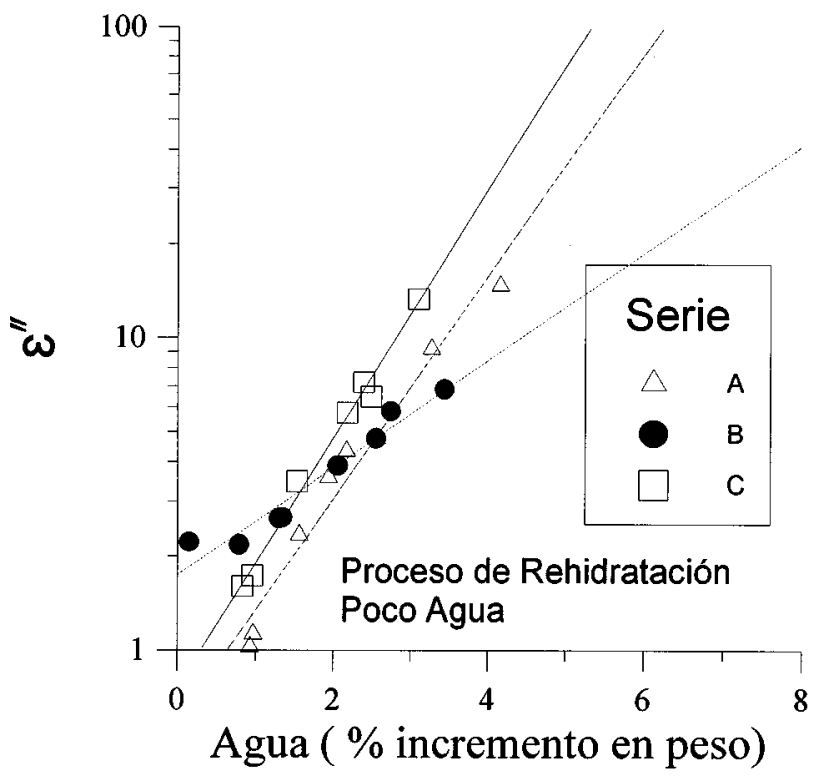

Figura 5. Variación con el contenido de agua a $1 \mathrm{kHz}$, de la parte imaginaria de la constante dieléctrica $\left(\varepsilon^{\prime \prime}\right)$ dos familias de poros, pero de tamaños considerablemente menores, prácticamente un orden de magnitud, e invirtiendo la relación, puesto que la porosidad es mayor en los de mayor diámetro medio.

La medida del nivel de rehidratación de muestras de los diferentes procesados, pone de manifiesto, que inicialmente a igual relación a/c, varía más rápidamente el contenido de agua en las muestras con cloruros, y con el mismo nivel de cloruros, lo hacen las muestras con mayor contenido de agua. Estas diferencias, pueden justificarse a partir de la Ley de Jourin. Esta ley permite estimar la altura que alcanza un líquido dentro de un capilar, $\left(\mathrm{h}_{\mathrm{c}}\right)$, en función de la Tensión Superficial, $\mathrm{T}_{\mathrm{S}^{\prime}}$ del ángulo del menisco, a, del radio medio de poro estimado, r, y de la densidad del líquido.

$$
h_{c}=\frac{2 \cdot T_{s} \cdot \cos \alpha}{\gamma \cdot r}
$$

Al tratarse del mismo líquido, y analizarse las muestras en las mismas condiciones, podemos asumir que todos los parámetros son iguales, salvo el radio medio de poro. Como éste es considerablemente menor en las muestras con cloruros, el nivel de agua en el interior en condiciones iguales, es mayor. Por su parte, las muestras sin cloruros, presentan diámetros de poro similares, pero al existir una mayor porosidad en las muestras A, su nivel de agua, y la velocidad de variación es mayor que en las muestras C. Por otra parte, el nivel de saturación se alcanza antes en estas últimas muestras, porque al ser menos porosas, admiten menor cantidad de agua. Así, mientras en las muestras B, con cloruros, se alcanzan niveles de contenido en agua de hasta $25 \%$ en peso, y en muestras A, estos valores son del $21 \%$, en las muestras $C$ difícilmente, se superan valores de aumento en peso del $11 \%$. (figura 1)

Se han caracterizado eléctricamente muestras de las tres series en todas las etapas del proceso de rehidratación. Los resultados que presentamos a continuación, corresponden a las primeras etapas del proceso. En esta etapa, es de esperar la contribución de dos elementos a la variación de la respuesta eléctrica de las muestras. Por una parte, la presencia de agua en los poros, y por otra la posible entrada de agua en la estructura del material. En una primera aproximación a separar ambos fenómenos, podemos suponer $(6,8)$ que la presencia de agua en los poros, contribuirá fundamentalmente a modificar la conductividad del material, y en consecuencia, afectará fundamentalmente a las variaciones o bien de parte real de la impedancia, o de la parte imaginaria de la constante dieléctrica. Por su parte, al ser el hormigón un dieléctrico, la entrada de agua en la estructura del mismo, debería afectar en mayor medida a la parte imaginaria de la impedancia (asociada en este caso a la capacidad), o a la parte real de la constante dieléctrica. Teniendo presente este planteamiento, nos centraremos en el análisis de estos parámetros. Las figuras 2, 3, 4 y 5 recogen el comportamiento para bajos contenidos de agua de $R, X, \varepsilon^{\prime}$ y $\varepsilon^{\prime \prime}$. Del análisis de estos resultados, se desprende que el comportamiento es mas parecido cuando la variación introducida en las muestras es la relación agua/cemento, que cuando se varía el contenido de cloruros. Este resultado era de esperar puesto que al añadir cloruros, se varía fuertemente la capacidad de captación de agua de la matriz del hormigón, y especialmente, se aportan elementos iónicos, que favorecen considerablemente la conducción en el interior del material. Este hecho se pone de manifiesto, en todas las gráficas, pero especialmente en la de R y la $\varepsilon^{\prime \prime}$.

Teniendo presente que nuestro objetivo es determinar los 


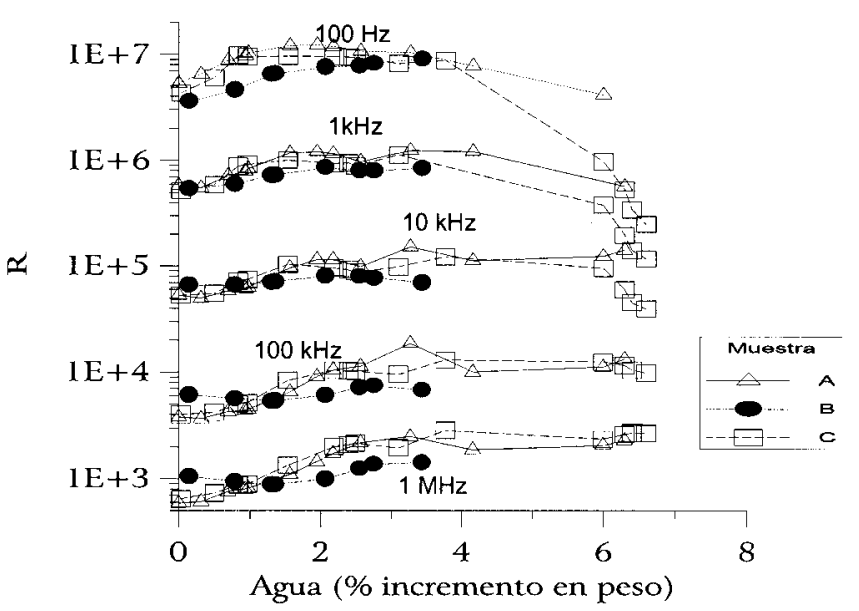

Figura 6. Variación de $\mathrm{R}$ con el contenido de agua para diferentes frecuencias de trabajo

procesos implicados en la rehidratación del material, es muy significativo el resultado reflejado en las medidas experimentales. En la figura 2 se observa un considerable aumento de la resistencia en el material cuando se inicia la entrada de agua en el mismo, hasta valores, que dependiendo del tipo de procesado se encuentran entre el 2 y el $4 \%$ de incremento en peso. Si completamos esta información analizando las curvas de la parte real e imaginaria de la permitividad dieléctrica, se comprueba que en todo el rango de trabajo, los valores de $\varepsilon^{\prime}$ son considerablemente mayores que los de $\varepsilon^{\prime \prime}$. Ambos parámetros evolucionan de forma similar, con un aumento permanente en el caso de muestras sin cloruros, y con una inflexión en las muestras con cloruros, que se prolonga hasta valores más altos de contenido de agua para $\varepsilon^{\prime}$.

Del análisis pormenorizado de estos resultados, puede desprenderse, que en la primera etapa de los procesos de rehidratación, el agua que entra en el material, pasa en su mayor parte a la estructura interna del mismo, hasta un nivel mínimo, a partir del cual, el proceso que es predominante es el de retención de agua en los poros. Este valor mínimo, está directamente relacionado con el tipo de material, y es menor, cuanto menor es la relación a/c del material, y cuanto menor sea el contenido de cloruros.

Aparentemente, este resultado es contradictorio, puesto que un material con menor relación a/c, debería ser más propenso a introducir agua en su estructura interna. Sin embargo, como hemos comentado anteriormente, una menor relación a/c, implica una reducción drástica del nivel de porosidad, por lo que con niveles de agua relativamente menores, se produce la saturación de la estructura interna del material en las regiones accesibles al agua. Por eso, inicialmente su variación es más fuerte, pero queda truncada para valores de contenido de agua menores.

El rango de frecuencias en que se ha analizado el comportamiento de estas muestras, es de $20 \mathrm{~Hz}$ a $1 \mathrm{MHz}$. En la figura 6, se muestra la evolución de la parte real de la impedancia para diversas frecuencias de trabajo. Puede observarse, que la fuerte inflexión presente a bajas frecuencias, se reduce a medida que nos aproximamos a los $\mathrm{MHz}$. Este resultado, refuerza las conclusiones anteriores. A bajas frecuencias, el comportamien- to resistivo del material está asociado fundamentalmente a la movilidad de cargas, mientras que a altas frecuencias lo que predomina es el comportamiento capacitivo del mismo. El aumento permanente de la impedancia con el contenido de agua, para frecuencias altas, pone de manifiesto la entrada de éste en la estructura del material, y en consecuencia, varía su comportamiento capacitivo, manteniéndose prácticamente estable para valores superiores al crítico (2-4\%) en lugar de disminuir como sucedía para frecuencias menores.

\section{CONCLUSIONES}

Se ha analizado el proceso de rehidratación de hormigones, diferenciando los procesos de absorción de agua implicados. De los resultados obtenidos, se concluye, que en una primera etapa la entrada de agua en el material, se produce en la estructura interna del mismo, hasta alcanzar un valor crítico, que dependiendo de las características de procesado del material varía entre el 2 y el $4 \%$ de incremento en peso. A partir de este valor, el factor predominante es el de retención de agua en los poros del material.

Comparando los diferentes procesados, las muestras con cloruros adquieren el nivel crítico para contenidos de agua mayores.

Por su parte las muestras sin cloruros, presentan el nivel crítico más alto para relaciones a / c mayores. Este resultado aparentemente contradictorio (al tener menor proporción de agua en la etapa de formación del material debería haber una mayor avidez del mismo), se justifica basándose en la diferente porosidad de las muestras. Menor relación a/c implica niveles de porosidad considerablemente menores. Esto hace que con menos agua se consiga la saturación de todo el material al que es capaz de acceder el agua, sin posibilidad de acceso a regiones interiores, potencialmente ávidas de agua.

\section{AGRADECIMIENTOS}

Este trabajo ha sido financiado a través de la CICYT con el proyecto MAT97-0694-C02.

\section{BIBLIOGRAFÍA}

1. N.R. Buenfeld, J.B. Newman and C.L. Page. The resistivity of mortars inmersed in sea-water. Cem. Concr. Res. 16, 511-524 (1986).

2. P.R. Camp and S. Billota. Dielectric properties of portland cement pasta as a function of tiem since mixing. J. Appl. Phys, 35, 1125-1131 (1990)

3. J.M. Tobio. Tiempo de fraguado del cemento. Examen crítico de algunos métodos para su determinación. Cemento y Hormigón, 325, 170-180 (1961)

4. J. Paquet. Mesure d humidité in'situ par les méthodes diélectriques application au betón. Matériaux et Constructions, 4, 20, 87-100 (1971).

5. M. Pérez-Pena, P.M. Roy, A.S. Bhalla and L.E. Cross, Dirlectric properties of densified hardened cementitious materials. Cem. Concr. Res., 16, 951-965 (1986).

6. C. Andrade, L. Soler, J.de Frutos.. Propiedades dieléctricas de pastas de cemento con bajo contenido de agua libre. Bol. Soc. Esp. Cerám. Vidrio, 34 5-6, 414-417 (1995)

7. E. Gayo, J. de Frutos, A. Palomo and S. Massa. A mathematical model simulating the evaporation processes in building materials: Experimental checking trough infrared thermography. Building and Environment, 31, 5, 477486 (1996)

8. E. Gayo, J. de Frutos. Interferential filters as an enhacement tool of infrared thermography in humidity studies. Infr. Phys. Tech., 38, 251-258, (1997) 